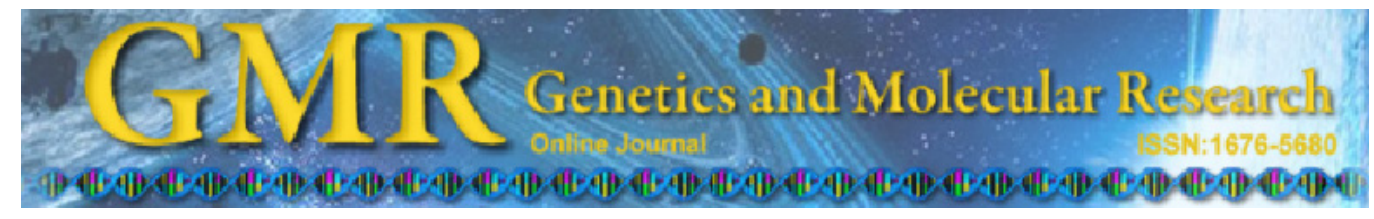

\title{
Cytogenetics of the darkling beetles Zophobas aff. confusus and Nyctobates gigas (Coleoptera, Tenebrionidae)
}

\author{
A.C. Lira-Neto ${ }^{1,3}$, G.M. Silva ${ }^{1}$, R.C. Moura ${ }^{2}$ and M.J. Souza ${ }^{1}$ \\ ${ }^{1}$ Departamento de Genética, Centro de Ciências Biológicas, \\ Universidade Federal de Pernambuco, Recife, PE, Brasil \\ ${ }^{2}$ Departamento de Biologia, Instituto de Ciências Biológicas, \\ Universidade de Pernambuco, Recife, PE, Brasil \\ ${ }^{3}$ Instituto Agronômico de Pernambuco, Recife, PE, Brasil \\ Corresponding author: R.C. Moura \\ E-mail: ritamoura.upe@gmail.com
}

Genet. Mol. Res. 11 (3): 2432-2440 (2012)

Received January 20, 2012

Accepted May 26, 2012

Published June 15, 2012

DOI http://dx.doi.org/10.4238/2012.June.15.5

ABSTRACT. Males of Zophobas aff. confusus and Nyctobates gigas (Tenebrionidae) collected in the State of Pernambuco, Brazil, were studied through conventional staining, C-banding, silver nitrate impregnation $\left(\mathrm{AgNO}_{3}\right)$, and the base specific fluorochromes $\mathrm{CMA}_{3}$ and DAPI. Z. aff. confusus was found to have $2 \mathrm{n}=20(9+\mathrm{Xyp})$ while $N$. gigas exhibited $2 \mathrm{n}=18(8+$ neoXY). Large pericentromeric blocks of constitutive heterochromatin $(\mathrm{CH})$ were detected throughout the autosomal complement of the two species, except in one autosomal pair of $N$. gigas in which no heterochromatic block was observed. The sex chromosomes of both species were almost totally heterochromatic. Double staining with $\mathrm{CMA}_{3} / \mathrm{DA}$ (distamycin) and DAPI/DA marked $\mathrm{CH}$ in $Z$. aff. confusus. However, DAPI staining was more intense. $N$. gigas was found to possess blocks of $\mathrm{CH}$-positive $\mathrm{CMA}_{3}$ and homogeneous DAPI. $\mathrm{AgNO}_{3}$ staining also revealed differences between the two species. In $Z$. confusus an NOR was observed in the sexual bivalent Xyp and N. gigas was found to have an autosomal NOR.

Key words: Beetle; Karyotype; Heterochromatin; Fluorochrome; NOR 


\section{INTRODUCTION}

The order Coleoptera, the largest order of the animal kingdom, comprises about 25,368 genera and 357,899 species. The Tenebrionidae family (suborder: Polyphaga) includes about 20,000 species that are distributed worldwide. Thus far, approximately 478 genera and 4624 species belonging to the Tenebrionidae family, of which 147 genera and 1234 species have been registered in Brazil, have been reported in the neotropical region (Costa, 2000).

Thus far, more than $1 \%$ of tenebrionids have been cytogenetically studied. The diploid number of tenebrionids ranges from $2 \mathrm{n}=14$ (Diaperis boleti and Scotobius miliaris) to $2 \mathrm{n}=38$ (Blaps gibba), with a modal karyotype containing 20 chromosomes $(2 \mathrm{n}=20)$. Although this species is frequently characterized by the $X_{y_{p}}$ sex system, other simple systems $\left(\mathrm{XY}, \mathrm{X} 0\right.$, and $\left.\mathrm{Xy}_{\mathrm{r}}\right)$ and multiple $\left(\mathrm{X}_{1} \mathrm{X}_{2} \mathrm{Y}, \mathrm{X}_{1} \mathrm{X}_{2} \mathrm{X}_{3} \mathrm{Y}, \mathrm{X}_{1} \mathrm{X}_{2} \mathrm{X}_{3} \mathrm{X}_{4} \mathrm{Y}\right.$, and $\left.\mathrm{X}_{1} \mathrm{X}_{2} \mathrm{X}_{3} \mathrm{X}_{4} \mathrm{Y}_{1} \mathrm{Y}_{2}\right)$ sex chromosomes have also been found (Juan and Petitpierre, 1991; Vitturi et al., 1996; Pons, 2004). The chromosomal morphology is generally metacentric and submetacentric, with chromosomes having similar sizes, which renders the identification of individual species difficult (Petitpierre, 1996). Some studies have focused on the distribution and base pair composition of the constitutive heterochromatin in the karyotypes of Tenebrionidae species (Juan and Petitpierre, 1989; Almeida et al., 2000; Pons, 2004). In general, the heterochromatin has a pericentromeric location and accounts for about $50 \%$ of the genome and constitutes monomeric sequences with varying sizes of 100-360 base pairs (Mestrovic et al., 2000). These sequences are primarily AT rich (Pons et al., 1993; Juan et al., 1993; Plohl et al., 1993; Ugarkovic et al., 1994). Studies on the nuclear organizing regions (NORs) and chromosome mapping studies are still quite scarse in this group (Juan et al., 1993; Vitturi et al., 1996).

In this study, the mitotic and meiotic chromosomes of Nyctobates gigas and Zophobas aff. confusus were analyzed using conventional staining, C-banding, base-specific fluorochrome staining, and silver nitrate impregnation. The results might contribute to the understanding of chromosomal diversification patterns in the family Tenebrionidae.

\section{MATERIAL AND METHODS}

The meiotic and mitotic chromosomes of 5 male individuals of $N$. gigas and 15 male individuals of $Z$. aff. confusus (Tenebrionidae) were analyzed. The individuals were collected from Tapacurá Biological Reserve in São Lourenço da Mata ( $\left.8^{\circ} 02^{\prime} 24 \mathrm{~S}: 35^{\circ} 11^{\prime} 45 \mathrm{~W}\right)$ and Vila Velha (748'34 S: 3451'26 W), Itamaracá, Pernambuco State, Brazil. The individuals were anesthetized with ether for the dissection of the testicular follicles; some individuals were treated with $0.2 \%$ colchicine for $6-9 \mathrm{~h}$. The testicles were fixed in ethanol:acetic acid (3:1). The cytological preparations were obtained by squashing the testicular follicles. For conventional staining, 2\% lacto-acetic orcein was used. The C-banding and double staining with $\mathrm{CMA}_{3} / \mathrm{DA}$ (distamycin) and DAPI/DA were performed according to Sumner (1972) and Schweizer et al. (1983), respectively, with minor modifications. For C-banding, the slides were treated with $0.2 \mathrm{~N} \mathrm{HCl}$ for $30 \mathrm{~min}$ at room temperature and $5 \%$ barium hydroxide for $30 \mathrm{~s}$ and $2 \mathrm{X} \mathrm{SSC}$ for $45 \mathrm{~min}$ at $60^{\circ} \mathrm{C}$. For $\mathrm{CMA}_{3} / \mathrm{DA}$ double staining, the slides were stained with $\mathrm{CMA}_{3}$ for $1 \mathrm{~h}$ and DA for $40 \mathrm{~min}$. For DAPI/DA staining, the slides were treated 
for 20 min with DAPI and 40 min with DA. Silver nitrate impregnation was performed as described by Rufas et al. (1987). In brief, the slides were pre-treated with $2 \mathrm{X} \mathrm{SSC}$ at $60^{\circ} \mathrm{C}$ for $10 \mathrm{~min}$ and stained with silver nitrate $(1 \mathrm{~g} / \mathrm{mL})$ at $70^{\circ}-80^{\circ} \mathrm{C}$.

The cells were analyzed and photographed using a Leica DMLB microscope. For conventional staining, Kodak Imagelink Wing 25 film was used for C-banded and silver nitrate-stained slides, and Kodak T-MAX WING 400 film was used for cells stained with $\mathrm{CMA}_{3} / \mathrm{DA}$ and DAPI/DA. The cell images were printed using Kodak Kodabrome Print F3.

\section{RESULTS}

The 2 species studied had differences in the diploid number and sex chromosome bivalent. Spermatogonial metaphase analysis allowed the identification of the number and precise chromosomal morphology of both the species. $Z$. aff. confusus had a diploid number of $2 \mathrm{n}=20$ (Figure 1a), with meioformula $9+\mathrm{Xy}_{\mathrm{p}}$ (Figure 1c), while $N . \operatorname{gigas}$ had $2 \mathrm{n}=18$ (Figure 1b), with meioformula $8+$ neoXY (Figure 1d). The karyotype of $Z$. aff. confusus consisted of metacentric and submetacentric chromosomes having discrete size differences. In $Z$. aff. confusus, the $\mathrm{X}$ chromosome is metacentric, while the y chromosome is puntiform (Figure 1a, c). This pattern is independent of the occurrence of NORs in Xyp, and it can result in the presence of argyrophilic substances in the lumen of that sex bivalent. The argyrophilic substances are important for the parachute configuration and segregation of the Xyp chromosomes. $N$. gigas had a karyotype comprising chromosomes decreasing gradually in size: 3 metacentric pairs, 3 submetacentric pairs, and 2 acrocentric pairs. In N. gigas, the neoX is submetacentric, while the neoY is acrocentric (Figure $1 \mathrm{~b}$ and d). The metaphase I of $Z$. aff. confusus showed a parachute type sex-determining system, with a metacentric X chromosome and diminute y chromosome (Figure 1c). In N. gigas, heteromorphism was observed in the sex chromosome neoXY, because these elements were unpaired due to the presence of only one chromosomal arm (Figure 1d). C-banding revealed constitutive heterochromatic distribution pattern, with large blocks of heterochromatin located in the pericentromeric region of all the chromosomes of $Z$. aff. confusus (Figure 2a and b) and $N$. gigas (Figure $3 \mathrm{a}$ and $\mathrm{b}$ ). The sex chromosomes of the 2 species were almost completely heterochromatic, except the $\mathrm{y}_{\mathrm{p}}$ of $Z$. aff. confusus.

Double staining with $\mathrm{CMA}_{3} / \mathrm{DA}$ and DAPI/DA revealed different patterns for the $\mathrm{CH}$ base pair composition in $Z$. aff. confusus and $N$. gigas. In $Z$. aff. confusus, $\mathrm{CMA}_{3}$ and DAPI stained the same regions of $\mathrm{CH}$ as those stained by $\mathrm{C}$-banding (Figure 2e and $\mathrm{f}$ ). $N$. gigas exhibited strong positive $\mathrm{CMA}_{3}$ markers in all chromosomes of the complement, except in 1 autosomal pair, indicating a CG-rich $\mathrm{CH}$ (Figure 3e and f). DAPI staining was homogeneous in all the chromosomes of $N$. gigas (Figure $3 \mathrm{~g}$ ).

Silver nitrate impregnation also revealed distinct patterns for the 2 studied species. In $Z$. aff. confusus, an amorphous mass, corresponding to the nucleolus, associated with the $\mathrm{Xy}_{\mathrm{p}}$ bivalent was noted (Figure 2c). In metaphase I, the sex bivalent showed $\mathrm{AgNO}_{3}$-stained parachute lumen (Figure 2d). In N. gigas, a strong impregnation was observed in an autosomal bivalent (Figure 3c). In both the species, $\mathrm{AgNO}_{3}$ also stained the regions corresponding to the $\mathrm{CH}$ (Figures $2 \mathrm{c}$ and $\mathrm{d}, 3 \mathrm{c}$ and $\mathrm{d}$ ). 


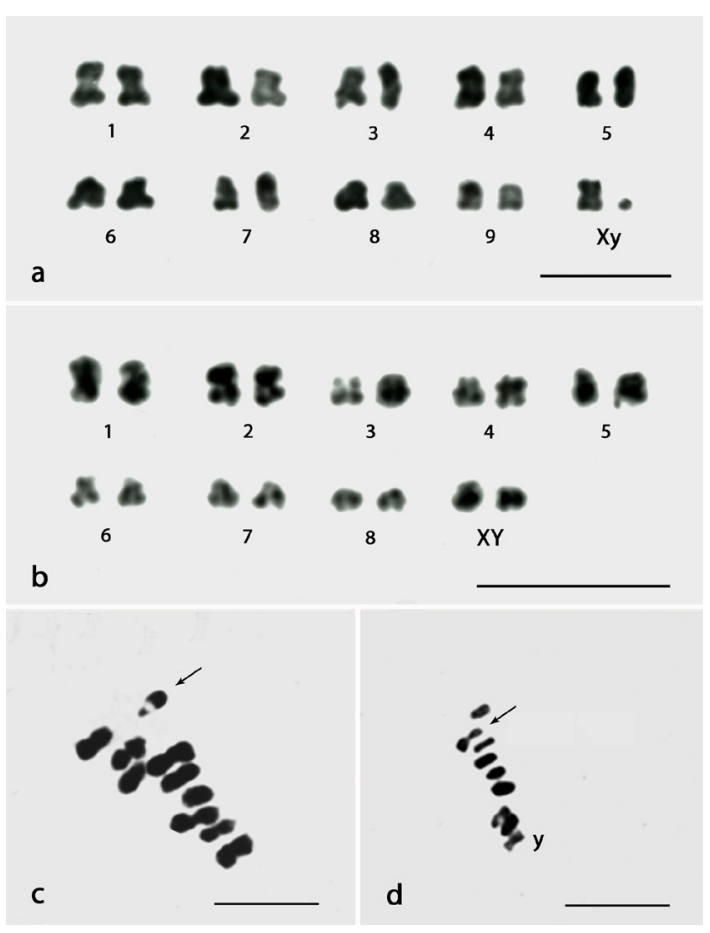

Figure 1. Spermatogonial metaphases and cells in metaphase I stained conventionally in Zophobas aff. confusus (a, c) and Nyctobates gigas $(\mathbf{b}, \mathbf{d})$. Bar $=3 \mu \mathrm{m}$.

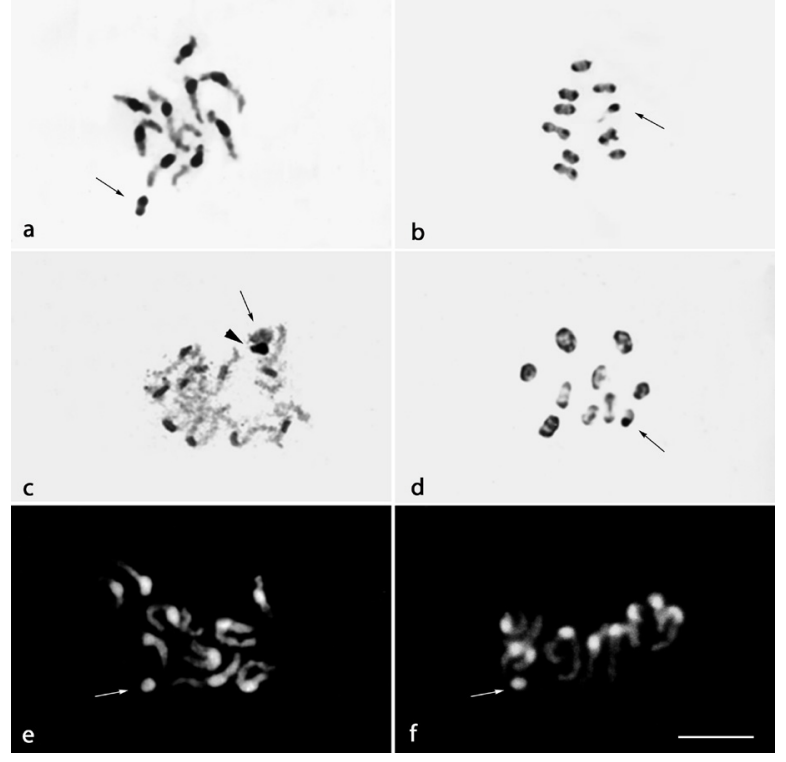

Figure 2. Meiotic cells of Zophobas aff. confusus after C-banding (a, b), silver nitrate impregnation (c, d), $\mathrm{CMA}_{3}$ (e), and DAPI (f). Pachytene cells (a, c, e, f) and cells in metaphase I (b, d). The arrows indicate Xyp. The arrowhead in $\mathbf{c}$ indicates NOR. Bar $=3 \mu \mathrm{m}$. 


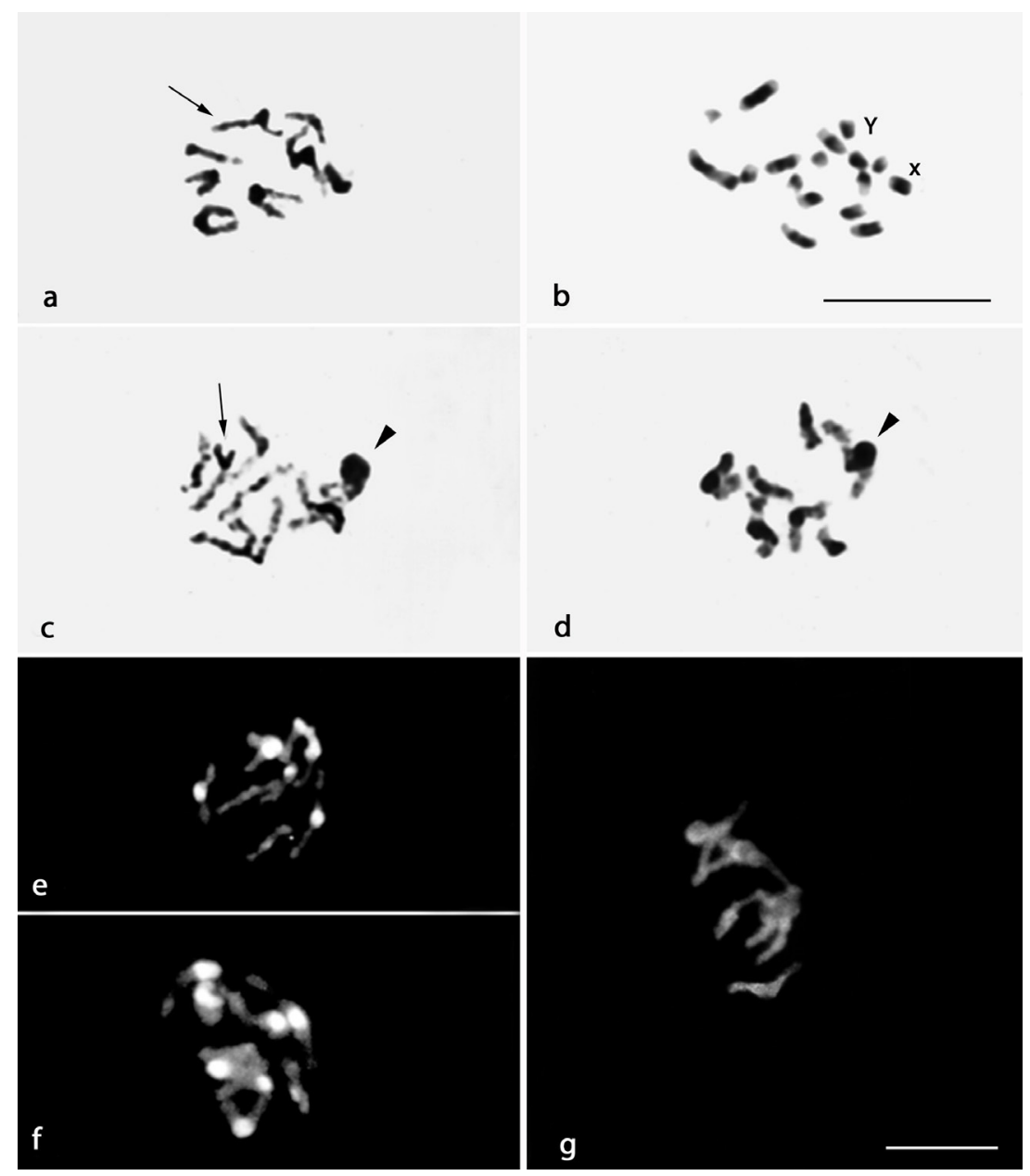

Figure 3. Meiotic and mitotic cells of Nyctobates gigas after C-banding (a, b), silver nitrate impregnation (c, d), $\mathrm{CMA}_{3}(\mathbf{e}, \mathbf{f})$, and DAPI $(\mathbf{g})$. Pachytene cells $(\mathbf{a}, \mathbf{c}, \mathbf{d}, \mathbf{e}, \mathbf{f}, \mathbf{g})$ and spermatogonial metaphase (b). The arrows in $\mathbf{a}$ and $\mathbf{c}$ indicate the bivalent neoXY. The arrowheads in $\mathbf{c}$ and $\mathbf{d}$ point to nuclear organizing region. The bar in $\mathbf{g}$ serves for all figures except figure $\mathbf{b}$. $\mathrm{Bar}=3 \mu \mathrm{m}$.

\section{DISCUSSION}

In general, the species belonging to the order Coleoptera have a high degree of conservation with regard to the diploid number $(2 \mathrm{n}=20)$, chromosomal sex system $\left(\mathrm{Xy}_{\mathrm{p}}\right)$, and meta-submetacentric chromosomes. However, numeric and morphologic variation in chromosomes of different taxa, for example, in families and subfamilies, have been described in the literature. Different structural rearrangements such as Robertsonian rearrangements and in tandem fusions and fissions can be responsible for such variations (Smith and Virkki, 1978; Vidal and Nocera, 1984; Petitpierre et al., 1991; Schneider et al., 2006; Moura et al., 2008; Cabral-de-Mello et al., 2008). 
The 2 species described herein differed with regard to the diploid number, chromosomal morphology, and sex chromosome bivalent. The karyotype of $Z$. aff. confusus consisted of 20 meta-submetacentric chromosomes that were similar in size and an $\mathrm{Xy}_{\mathrm{p}}$ sex system, with a small yp. This was similar to the modal karyotype for the Coleoptera order (Smith and Virkki, 1978), which has been reported in $63 \%$ of the Tenebrionidae species studied thus far (Juan and Petitpierre, 1991). N. gigas had a distinct karyotype unlike the modal and primitive karyotypes for Coleoptera; it had a diploid number of $2 \mathrm{n}=18$ and a derived sex system-neoXY, along with 2 pairs of acrocentric chromosomes. The smaller diploid number could possibly result from an X-autosome fusion, responsible for the origin of the neoXY sex system. The neoXY sex system has been frequently reported in the representatives of the tribe Akidini. The presence of this sex system in the Tenebrionidae family might be an exceptional case since all the Tenebrionidae species studied have a $7+$ neoXY sex system (Juan and Petitpierre, 1991). Reduction in the diploid number has been reported in N. gigas and representatives of tribe Akidini (genus Pimelia (Pimeliini)), although this process did not involve sex chromosomes, and the ancestral Xyp sex system was maintained. The ancestral species belonging to genus Pimelia have a reduced diploid number of $8+$ Xyp (Pons, 2004).

In Coleoptera, the neoXY sex system originated by chromosomal rearrangements among the ancient Xyp or XO sex system and autosomal elements. In this group, the presence of the neoXY system is more common in representatives of families such as Carabidae and Passalidae that have a higher prevalence of the XO system (Smith and Virkki, 1978). Therefore, the origin of the neoXY system in the tenebrionid Pimelia sparsa sparsa $(8+$ neoXY) has been thought to result from a rearrangement involving the ancient Xyp with loss of most of the existent heterochromatin in the X chromosome (Pons, 2004). Schneider et al. (2006) also suggested that the fusion of autosomal/Xy chromosome might be responsible for the origin of the $7+$ neoXY karyotype in Conoderus stigmosus (Elateridae).

$Z$. aff. confusus and $N$. gigas showed a similar pattern of $\mathrm{CH}$ distribution in the pericentromeric regions of the autosomes. In addition, the neoX and neoY chromosomes of $N$. gigas and the $\mathrm{X}$ chromosome of $Z$. aff. confusus were almost completely heterochromatic. A similar pattern of heterochromatic $\mathrm{X}$ chromosome has been described in most beetles such as Gonocephalum patruele (Tenebrionidae) and Harpalus affinis (Carabidae) and in some species belonging to the family Scarabaeidae (Juan and Petitpierre, 1989; Rozek and MaryanskaNadachowska, 1991; Moura et al., 2003; Bione et al., 2005a,b). However, some species of the order Coleoptera have been described to have a euchromatic X chromosome, such Trechus latus (Carabidae) (Rozek, 1998). In addition, as observed in Z. aff. confusus, the presence of euchromatic y has been described in Gymnopleurus sturmi (Scarabaeidae), Palembus dermestoides (Tenebrionidae), Epicauta atomaria (Meloidae), and in 23 of the 25 studied species of Pimelia (Tenebrionidae) (Almeida et al., 2000; Colomba et al., 2000; Pons, 2004). In these species, the lack of labeling can be due to the minuscule size of the $\mathrm{CH}$ blocks in the yp. Juan et al. (1993) conducted FISH studies and showed the presence of repetitive DNA in the yp chromosome of some species of Coleoptera.

The larger amount of $\mathrm{CH}$ found in the species studied herein represents an important characteristic of Tenebrionidae species. In most of the species in which the heterochromatin was quantified, the values reached almost $50 \%$ of the genome content (Juan and Petitpierre, 1989; Plohl et al., 1993; Pons et al., 1997; Pons, 2004; Mravinac et al., 2004). Such a CH distribution pattern has rendered these organisms to be used as model systems for studying the structure, function, and evolution of $\mathrm{CH}$ and their components. 
The use of base-specific fluorochromes revealed interspecific variation in the base pair composition of the $\mathrm{CH}$ of the 2 species studied here. Although the $\mathrm{CH}$ blocks were stained by both the fluorochromes in $Z$. aff. confusus, the DAPI labeling was stronger $\left(\mathrm{DAPI}^{++}\right)$than $\mathrm{CMA}_{3}$ labeling $\left(\mathrm{CMA}_{3}^{+}\right)$, indicating a predominance of AT. Study of the composition of $\mathrm{CH}$ by using fluorochromes and analysis of tandemly repeated DNA sequences this $\mathrm{CH}$ has revealed the presence of AT-rich blocks in all the analyzed species of Tenebrionidae (Juan et al., 1993; Plohl et al., 1993; Pons et al., 1993; Ugarkovic et al., 1994; Pons et al., 1997; Mestrovic et al., 2000). However, in N. gigas, a distinct pattern with CMA3+ heterochromatic blocks was observed, indicating the prevalence of GC base pairs. A similar pattern has been reported in other species such as Thorectes intermedius (Geotrupidae), Phyllophaga (Phyllophaga) aff. Capillata, and G. sturmi (Scarabaeidae) (Vitturi et al., 1999; Colomba et al., 2000; Moura et al., 2003).

Previous studies have suggested that nucleolus organization by 1 autosomal pair is responsible for the pattern of NOR location in Coleoptera (Drets et al., 1983; Virkki, 1983; Postiglioni and Brum-Zorrilla, 1988; Rozek, 1998; Colomba et al., 2000; Moura et al., 2003; Bione et al., 2005a). This pattern was also observed in N. gigas. However, Z. aff. confusus showed an active NOR associated with the Xyp bivalent. These NOR regions, also positive for $\mathrm{C}$-banding, stained brilliantly with $\mathrm{CMA}_{3}$, indicating that the whole heterochromatin stained with silver nitrate is rich in GC base pairs. In $Z$. aff. confusus, the nucleolar remnant is present until the end of pachytene, while Xyp remains positive for silver nitrate staining until metaphase I. Such a pattern has also been observed in some coleopteran species independent of NOR, irrespective of whether it is associated with Xyp (Postiglioni and Brum-Zorrilla, 1988; Moura et al., 2003; Bione et al., 2005b). This pattern is independent of the occurrence of NORs in the Xyp and can result in the presence of argyrophilic substances in the lumen of that sex bivalent. The argyrophilic substances are important in the parachute configuration and segregation of the Xyp chromosomes (Virkki et al., 1990, 1991).

Although the 2 species studied here belonged to the same family, subfamily, and tribe, their karyotypes had distinct features with regard to the chromosomal number, sexual system, $\mathrm{CH}$ composition, and NOR location. The results obtained in $Z$. aff. confusus are in agreement with those obtained for other species belonging to Tenebrionidae. However, the karyotype of $N$. gigas differed distinctly from the most common chromosomal patterns reported for Tenebrionidae, such as the diploid number, sex chromosome bivalents (18, neoXY), and base pair richness of the heterochromatin. Despite a conserved chromosomal pattern, Tenebrionidae possesses some tribes and genera that have derived chromosomal characteristics, indicating distinct chromosomal evolution trends in this group. Moreover, our results warrant the need

of studies involving a larger number of Tenebrionidae representatives for better understanding the evolution karyotype of this group.

\section{ACKNOWLEDGMENTS}

We thank Dr. Carlos Campaner, curator of the Museum of Zoology, Universidade de São Paulo (USP), for the taxonomic identification of the studied species and Francisca Tavares of Lira and Cirlene Maria da Silva for the technical support. We also thank Dr. Diogo Cavalcanti Cabral-de-Mello (UNESP Rio Claro) for the critical comments on the manuscript. Research supported by Conselho Nacional de Desenvolvimento Cientifico e Tecnológico (CNPq) and Fundação de Amparo à Ciência e Tecnologia do Estado de Pernambuco (FACEPE). 


\section{REFERENCES}

Almeida MC, Zacaro AA and Cella DM (2000). Cytogenenetic analysis of Epicauta atomaria (Meloidae) and Palembus dermestoides (Tenebrionidae) with Xyp sex determination system using standard staining, C-bands, NOR and synaptonemal complex microspreaning techniques. Hereditas 133: 147-157.

Bione E, Camparoto ML and Zilá LP (2005a). A study of the constitutive heterochromatin and nucleolus organizer regions of Isocopris inhiata and Diabroctis mimas (Coleoptera: Scarabaeidae, Scarabaeinae) using C-banding, $\mathrm{AgNO}_{3}$ staining and FISH techniques. Genet. Mol. Biol. 28: 111-116.

Bione E, Moura RC, Carvalho R and Souza MJ (2005b). Karyotype, C-and fluorescence banding pattern, NOR location and FISH study of five Scarabaeidae (Coleoptera) species. Genet. Mol. Biol. 28: 376-381.

Cabral-de-Mello DC, Oliveira SG, Ramos ICG and Moura RC (2008). Chromosome differentiation patterns in Scarabaeinae subfamily (Coleoptera: Scarabaeidae). Micron 39: 1243-1250.

Colomba MS, Vitturi R and Zunino M (2000). Karyotype analysis, banding, and fluorescent in situ hybridization in the scarab beetle Gymnopleurus sturmi McLeay (Coleoptera Scarabaeoidea: Scarabaeidae). J. Hered. 91: 260-264.

Costa C (2000). Estado de Conocimiento de los Coleoptera neotropicales. In: Hacia un proyecto CYTED para el inventario y estimación de la diversidad entomológica en Iberoamérica: PriBES-2000 (Martín-Piera F, Morrone JJ and Melic A, eds.). Sociedad Entomológica Aragonesa (SEA), Madrid, 99-114.

Drets ME, Corbella E, Panzera F and Folle GA (1983). C-banding and non-homologous association II. The "parachute" Xyp sex bivalent and the behavior of heterocromatin segments in Epilachna paenulata. Chromosoma 88: 249-255.

Juan C and Petitpierre E (1989). C-banding and DNA content in seven species of Tenebrionidae (Coleoptera). Genome 32: 834-839.

Juan C and Petitpierre E (1991). Chromossome Number and Sex-Determining Systems in Tenebrionidae (Coleoptera). Advances in Coleopterology, 167-176.

Juan C, Pons J and Petitpierre E (1993). Localization of tandemly repeated DNA sequences in beetle chromosomes by fluorescent in situ hybridization. Chromosome Res. 1: 167-174.

Mestrovic N, Mravinac B, Juan C, Ugarkovic D, et al. (2000). Comparative study of satellite sequences and phylogeny of five species from the genus Palorus (Insecta, Coleoptera). Genome 43: 776-785.

Moura RC, Souza MJ, Melo NF and Lira-Neto AC (2003). Karyotypic characterization of representatives from Melolonthinae (Coleoptera: Scarabaeidae): karyotypic analysis, banding and fluorescent in situ hybridization (FISH). Hereditas 138: 200-206.

Moura RC, Melo NF and Souza MJ (2008). High levels of chromosomal differentiation in Euchroma gigantea L. 1735 (Coleoptera, Buprestidae). Genet. Mol. Biol. 31: 431-437.

Mravinac B, Plohl M and Ugarkovic D (2004). Conserved patterns in the evolution of Tribolium satellite DNAs. Gene 332: $169-177$.

Petitpierre E (1996). Molecular cytogenetics and taxonomy of insects, with particular reference to the Coleoptera. Int. J. Insect Morphol. Embryol. 25: 115-133.

Petitpierre E, Juan C and Alvarez-Fuster A (1991). Evolution of Chromosomes and Genome size in Chysomelidae and Tenebrionidae (Coleoptera). In: Advances in Coleopterology (Zunino M, Bellés X and Blas M, eds.). AEC, Barcelona, 129-144.

Plohl M, Lucijanic-Justic V, Ugarkovic D, Petitpierre E, et al. (1993). Satellite DNA and heterochromatin of the flour beetle Tribolium confusum. Genome 36: 467-475.

Pons J (2004). Evolution of diploid chromosome number, sex determining systems, and heterochromatin in Western Mediterranean and Canarian species of the genus Pimelia (Coleoptera: Tenebrionidae). J. Zool. Syst. Evol. Res. 42: 81-85.

Pons J, Petitpierre E and Juan C (1993). Characterization of the heterochromatin of the darkling beetle Misolampus goudoti: cloning of two satellite DNA families and digestion of chromosomes with restriction enzymes. Hereditas 119: 179-185.

Pons J, Bruvo B, Juan C, Petitpierre E, et al. (1997). Conservation of satellite DNA in species of the genus Pimelia (Tenebrionidae, Coleoptera). Gene 205: 183-190.

Postiglioni A and Brum-Zorrilla N (1988). Non-relationship between nucleolus and sex chromosomes system Xyp in Chelymorpha variabilis boheman (Coleoptera: Chrysomelidae). Genetica 77: 137-141.

Rozek M (1998). C-bands and NORs on chromosomes in four species of the genus Trechus Clairv. (Coleoptera, Carabidae). Caryologia 51: 189-194.

Rozek M and Maryanska-Nadachowska A (1991). Studies on C-banding Karyotype and Meiosis of Harpalus affinis (Shrank, 1781) (Coleoptera, Carabidae). Caryologia 44: 317-323. 
Rufas JS, Giménez-Ábian J, Suja JA and Garcia de La Veja C (1987). Chromosome organization in meiosis revelead by litht microscope analysis of silver-stained cores. Genome 29: 706-712.

Schneider MC, Almeida MC, Rosa SP, Costa C, et al. (2006). Evolutionary chromosomal differentiation among four species of Conoderus Eschscholtz, 1829 (Coleoptera, Elateridae, Agrypninae, Conoderini) detected by standard staining, C-banding, silver nitrate impregnation, and CMA3/DA/DAPI staining. Genetica 128: 333-346.

Schweizer D, Mendelack M, White MJ and Contreras N (1983). Cytogenetics of partenogenetic grasshopper Warramaba virgo and its bisexual relatives X. Pattern of fluorescent banding. Chromosoma 88: 227-236.

Smith SG and Virkki N (1978). Coleoptera. In: Animal Cytogenetics (John B, ed.). Borntraeger, Berlin, Stuttgart, 366.

Sumner AT (1972). A simple technique for demonstrating centromeric heterochromatin. Exp. Cell Res. 75: 304-306.

Ugarkovic D, Plohl M, Petitpierre E, Lucijanic-Justic V, et al. (1994). Tenebrio obscurus satellite DNA is resistant to cleavage by restriction endonucleases in situ. Chromosome Res. 2: 217-223.

Vidal OR and Nocera CP (1984). Citogenética de la tribu Eucranini (Coleoptera, Scarabaeidae). Estudios Convencionales y con Bandeo C. Physis 42: 83-90.

Virkki N (1983). Banding of Oedionychna (Coleoptera: Alticinae) chromosomes: C-and Ag-bandis. J. Agric. Univ. Puerto Rico 67: 221-225.

Virkki N, Mazzella C and Denton A (1990). Staining of substances adjacent to the Xyp sex bivalent of some weevil (Coleoptera: Curculionidae). J. Agric. Univ. Puerto Rico 74: 405-418.

Virkki N, Mazzella C and Denton A (1991). Silver staining of coleopteran Xyp sex bivalent. Cytobios 67: 45-63.

Vitturi R, Catalano E, Sparacio I, Colomba MS, et al. (1996). Multiple-chromossome sex systems in the darkling beetles Blaps gigas and Blaps gibba (Coleoptera, Tenebrionidae). Genetica 97: 225-233.

Vitturi R, Colomba MS, Barbieri R and Zunino M (1999). Ribosomal DNA location in the scarab beetle Thorectes intermedius (Costa) (Coleoptera: Geotrupidae) using banding and fluorescent in-situ hybridization. Chromosome Res. 7: 255-260. 